Leslie Hannah

\title{
Die Überlebenschancen der Großen
}

Im folgenden untersuchen wir die Entwicklung von Großunternehmen und gehen der Frage nach, ob eindeutige Faktoren angegeben werden können, die das Wachstum oder den Verfall des Leistungsvermögens solcher Firmen bestimmen. Dabei betrachten wir die Fallstudien der Unternehmenshistoriker mit gewissen Vorbehalten; sie haben zwar unsere Kenntnisse über die Lernfähigkeit von Organisationen erweitert, aber sie neigen auch dazu, die Unternehmenserfolge über die Maßen hervorzuheben und die Mißerfolge zu vernachlässigen. Darüber hinaus stellt sich die Frage, ob die aus der Untersuchung einzelner Unternehmensgeschichten gewonnenen Ergebnisse verallgemeinerungsfähig sind, und vollends fragwürdig wird es, wenn die Unternehmensberater behaupten, die einzig richtigen Rezepte für eine erfolgreiche Entwicklung der Firma gefunden zu haben.

Der englische Ökonom Alfred Marshall beschäftigte sich bereits sehr früh im Rahmen seiner Theorie der Unternehmung mit dem Lebenslauf der Firmen. Er schrieb in einer Zeit, in der Großunternehmen in einem bis dahin unbekannten Ausmaß entstanden. In der ersten Auflage seiner Principles of Economics aus dem Jahre 1890 formuliert er die These, daß sowohl die großen als auch die kleinen Unternehmen »allesamt früher oder später vom Alterungsprozeß erfaßt« würden, und vergleicht das Wachstum und den Verfall der Firmen mit dem Lebenslauf der Bäume im Wald. In der sechsten Auflage aus dem Jahre 1910 wurde Marshall jedoch etwas zweifelnd und veränderte seine Position, »weil große Aktiengesellschaften (...) zwar oft stagnieren, aber nicht so leicht sterben« (Marshall, Ausgabe von Guillebaud 1961, 316). Marshall war ein genauer Beobachter der realwirtschaftlichen Verhältnisse in Großbritannien, Deutschland und Amerika; er gelangte aufgrund seiner empirischen Einsichten zu dem Schluß, daß die Großunternehmen - die riesigen Redwood-Bäume, um im Bild zu bleiben -, die in der Zwischenzeit entstanden waren, sich gründlich von jenen Firmen des 19. Jahrhunderts unterschieden, die ihm zwei Jahrzehnte zuvor als Anschauungsobjekt für seine Theorie der Unternehmung dienten.

Die Untersuchungen von Unternehmenshistorikern - auch wenn sie im übrigen Marshalls Sichtweise der Industrieökonomik ablehnen - stimmen mit 
seinem Urteil überein, daß sich in den Industrieunternehmen des 20. Jahrhunderts eine bedeutsame Veränderung vollzog. So weisen Chandler (1990) und Lazonick (1991) darauf hin, daß zu Beginn dieses Jahrhunderts die Großunternehmen bedeutende technische und organisatorische Fähigkeiten ausbilden und ein leistungsfähiges Marketing entwickeln konnten, wodurch sie unangreifbare Vorreitervorteile erwarben, die es ihnen auch heute im allgemeinen noch erlauben, die globalen oligopolistischen Märkte zu dominieren, die sie selbst erst geschaffen haben. Im folgenden geht es nicht darum, die Position der genannten Autoren - Marshall eingeschlossen - in Zweifel zu ziehen, denn sie haben fraglos recht! Der vorliegende Aufsatz geht hingegen der Frage nach, welche der beiden Analogien die Realität der modernen, unternehmerisch verfaßten Wirtschaftssysteme besser zu beschreiben vermag: die traditionelle Analogie, die das Bild von zuerst wachsenden und dann absterbenden Bäumen zeichnet, oder die neue, welche die Großunternehmen den riesigen Redwoods gleichstellt und deren Lebensspanne nicht in Jahrzehnten, sondern in Jahrhunderten mißt. Unserer Auffassung nach ist die alte Sichtweise keineswegs obsolet geworden; sie kann unser Verständnis von den Eigenarten, den Stärken und den Schwächen fördern, die im Wandel des organisatorischen Leistungsvermögens der Unternehmen eingeschlossen sind.

\section{Methodische Überlegungen}

Betrachtet man allein den Umstand, daß zahlreiche Firmen, die heute die Märkte dominieren, bereits eine sehr lange Lebensgeschichte aufweisen oder daß einige der größten heutigen Firmen bereits vor hundert Jahren zur Spitze zählten, dann könnte sich der Schluß aufdrängen, daß die Großunternehmen im allgemeinen eine lange Lebensdauer haben. Diese Beobachtung ist aber andererseits auch mit der Hypothese vereinbar, daß einige der ursprünglich kleinen Firmen schnell wachsen, wohingegen Großunternehmen, über einen längeren Zeitraum betrachtet, eine sehr niedrige Überlebensrate aufweisen. Unser gegenwärtiges Wissen über die seit jener Zeit bestehenden Großunternehmen beherrscht unsere Vorstellung von typischen Unternehmensentwicklungen, und wir neigen dazu, die Erfolge der überlebenden Großunternehmen überzubetonen. Auf der anderen Seite wird die Geschichte der Mißerfolge vergessen oder als untypisch für die Unternehmensentwicklung behandelt.

Für die nachfolgende Untersuchung wurden die 100 größten, weltweit operierenden Industrieunternehmen des Jahres 1912 ausgewählt; wir verfolgen ihren Lebenslauf und gehen der Frage nach, wieviele von ihnen im Jahr 1995 noch zu den einhundert größten Unternehmen zählen (siehe auch Schmitz 1993). Die untersuchten Firmen enthalten beinahe alle Industrie- 
unternehmen (i.e. Bergbau und verarbeitendes Gewerbe) weltweit, deren Kapitalmarktwert im Jahr 1912 den Betrag von 26 Millionen Dollar erreichte oder überstieg. Selbst nach heutigen Maßstäben waren dies allesamt Großunternehmen: das größte Unternehmen (US Steel) hatte im Jahr 1912 221.025 Arbeiter; die typische Firma in dieser Liste beschäftigte mehr als 10.000 Arbeitskräfte. Im allgemeinen bestanden diese Unternehmen schon seit längerer Zeit und wurden im Durchschnitt bereits seit 32 Jahren als Aktiengesellschaften geführt; häufig waren sie noch älter und wurden als Genossenschaften oder frühe Personengesellschaften gegründet. Sie entstanden nicht aus den spekulativen Überhitzungen des Aktienmarkts, sondern überstanden den harten Ausleseprozeß der Fusionswellen und des Booms am Aktienmarkt um die Jahrhundertwende, in dem viele Großunternehmen mit einem geschwächten Kapitalwert und einer geringen Leistungsfähigkeit drastisch schrumpften (Livermore 1935, Lamoreaux 1984, Hannah 1974, Tilly 1982, Samber 1997). Im großen und ganzen wurden die ausgewählten Firmen von den zeitgenössischen Analysten aufgrund ihrer großzügigen und dauerhaften Dividendenzahlungen als attraktive und sichere Anlagen betrachtet (Meyer 1910, 196). Eine Gruppe von Großunternehmen aus dem Jahr 1900 würde sicherlich frühere Unternehmenszusammenbrüche und einen rascheren Verfall von Firmen aufweisen als unsere Gruppe aus dem Jahr 1912.

Wir benutzen die Kapitalmarktbewertung der Unternehmen in den Jahren 1912 und 1995, d.h. ihre jeweiligen Aktienwerte, um zu entscheiden, ob sie gewachsen sind und ihr Leistungsvermögen ausbauen konnten oder ob sie verfielen. Ein solcher Vergleich der Kapitalwerte von überlebenden Firmen muß die Inflation berücksichtigen. Um die Preisbereinigung durchzuführen, habe ich den Preisanstieg des US-amerikanischen Kapitalmarktes, genauer gesagt den »Standard \& Poor industrial 500 Index « benutzt. Der Preisanstieg am US-Aktienmarkt (der zwischen 1912 und 1995 durchschnittlich 6\% pro Jahr betrug) bringt zum Teil den Wertverlust des Dollar (durchschnittlich 4\% pro Jahr in diesem Zeitraum) und zum Teil das reale Wachstum zum Ausdruck. Es erscheint plausibel, einen Deflator zu benutzen, der auch den Umstand erfaßt, daß selbst solche Firmen, die ihre Marktposition gerade halten konnten, an diesem allgemeinen realwirtschaftlichen Wachstum teilhatten, das in den letzten einhundert Jahren im Durchschnitt aller OECD-Länder bei etwas mehr als $2 \%$ pro Jahr und pro Kopf lag, wobei auch zu berücksichtigen ist, daß die Zahl der Industriearbeiter angestiegen ist (Maddison 1989, 15). Auf dieser Grundlage können wir mit dem Quotienten aus dem Kapitalwert des Jahres 1995 (der in der angegebenen Weise deflationiert wird) und dem ursprünglichen Kapitalwert des Jahres 1912 eine Kennziffer erhalten, die im folgenden auch als Leistungsrate bezeichnet wird. 
Eine erste, intuitive Interpretation dieser Rate legt nahe, daß die Manager eines Unternehmens nichts verloren hätten, ausgenommen ihre Einkommen, wenn diese Rate eins betragen würde und die Kapitalwerte von 1912 und die deflationierten von 1995 gleich wären. Eine Rate kleiner als eins bedeutete, daß es vorteilhafter gewesen wäre, das Unternehmen 1912 aufzugeben; eine Leistungsrate größer als eins könnte nahelegen, daß die Fähigkeiten des Unternehmens erweitert (zum Beispiel durch räumliche Ausdehnung oder eine breitere Produktpalette) oder vertieft wurden (durch Forschung und Entwicklung oder die Einführung von Markenzeichen).

Indes sollte man mit solchen naheliegenden Schlußfolgerungen vorsichtig sein. Wäre ein Großunternehmen im Jahre 1912 verkauft worden, so hätte man nicht zwingend den Marktpreis realisiert: der Liquidationswert eines Unternehmens wäre geringer als sein Marktwert gewesen, Übernahmepreise hätten darüber liegen können. Ganz ähnlich sollte der Quotient aus den Kapitalwerten von 1995 und 1912 nicht als ein Maß für die Erträge der Investoren verstanden werden: letzteres erforderte eine Analyse der zwischenzeitlich gezahlten Dividenden (und anderer Ströme zwischen den Unternehmen und den Aktienbesitzern), die höher oder niedriger als der Standard \& Poor Index hätten sein können. Selbst wenn am Ende die Firma erloschen wäre, könnten die Ertäge für die Investoren noch höher sein, denn die Ausnutzung von Monopolgewinnen wird für sie in der Regel vorteilhafter sein als ein Restbestand an obsoletem Anlagevermögen. Außerdem könnte eine Firma, die unter Konkursschutz steht, die Vermögenswerte ihrer Kapitalanleger ernsthaft gefährden, und trotzdem groß bleiben, was eher ihr übriges Unternehmenspotential zum Ausdruck bringt als das zeitweilige finanzielle Mißmanagement oder einfach nur Pech. Unsere Aufmerksamkeit gilt jedoch nicht den Vorteilen der Investoren, sondern der Entfaltung oder dem Niedergang von Fähigkeiten, die in den Unternehmen verkörpert sind. $\mathrm{Zu}$ diesem Zweck interessieren wir uns für die Unternehmensgröße, soweit sie durch den Kapitalwert gemessen wird, und der bereinigte Kapitalwert des Jahres 1995 gibt darüber Auskunft, ob die Gesamtheit des Leistungsvermögens einer Firma gestiegen oder gesunken ist.

Selbstredend wurde die Unternehmensgröße neben dem Geschick, mit dem die Manager vormals die Aktiva des Unternehmens einsetzten, noch durch viele andere Faktoren beeinflußt. Die Marktposition und die Größe ermöglichten oft die Vorteile des Vorreiters, um sich aber in der Konkurrenz zu behaupten, kommt es nicht nur auf die Ausgangslage eines Unternehmens an, sondern auch auf die ständige Anpassung an die Marktbedingungen. Das Leistungsvermögen der Unternehmen änderte sich im Lauf der Zeit. Neue Ressourcen wurden dem Unternehmen durch Aktienemission oder durch die Übernahme anderer Firmen hinzugefügt. Aufgrund der Emission von Wertpapieren, die der Finanzierung von Unternehmensfusionen, der 
Akquisition anderer Unternehmen oder der internen Expansion dienten, haben sich die Aktienbesitzer wahrscheinlich schlechter gestellt als unser Maß für die Fähigkeiten des Managements nahelegt. Man sollte daher eine Leistungsrate von eins als ein schwaches und mit Vorbehalten versehenes Maß für die Schwelle betrachten, welche die Verbesserung der Leistungsfähigkeit eines Unternehmens festlegt.

Es bleiben zwei Probleme, um die Überlebensfähigkeit der Großunternehmen des Jahres 1912 zu beurteilen. Zum einen kann der Fall eintreten, daß der »Erbe« des ursprünglichen Unternehmens bestimmt werden muß, wenn nämlich letztere das Geschäftsfeld wechselte. Um Fragen der Abstammung eines Unternehmens zu klären, werden eher ökonomische als rechtliche Kriterien benutzt. Zum anderen muß man festlegen, wie mit denjenigen Großunternehmen verfahren werden soll, die von anderen übernommen wurden oder auf andere Weise zwischen 1912 und 1995 verschwunden sind. Wenn Unternehmenszusammenschlüsse rückgängig gemacht wurden, dann sehen wir von dieser Zwischenphase in der Unternehmensgeschichte $\mathrm{ab}$ : so ist Bayer der Nachfolger der Elberfelder Farbenfabriken und die zwischenzeitlich existierende IG Farben wird vernachlässigt. In allen Fällen, in denen eine freiwillige Entflechtung stattfand, betrachten wir das größere verbleibende Kerngeschäft als den Firmennachfolger. Wenn die Entflechtung jedoch auf staatliche Maßnahmen zurückgeht, dann werden die neu entstehenden Großunternehmen als ein kombinierter Nachfolger der urspünglichen Firma betrachtet.

Schließlich gibt es noch das Problem der Verstaatlichung, das insbesondere für die Großunternehmen aus dem Jahr 1912 ein ersthaftes Risiko darstellte. Alle russischen, mexikanischen und französischen Unternehmen auf der Liste von 1912, viele deutsche und Teile von britischen Firmen wurden zeitweise verstaatlicht und einige sind in staatlichem Besitz verblieben. Unternehmen wie BP und St. Gobain wurden zwar verstaatlicht, aber weiterhin wie private Firmen geführt, so daß hier keine methodischen Schwierigkeiten entstehen. Hingegen bereitet die Behandlung der riesigen russischen Firmen Schwierigkeiten, von denen es 1912 drei Stück gab. Das Unternehmen Nobel Brothers mit seinem Hauptsitz in St. Petersburg wurde nach der russischen Revolution enteignet und die ihm verbliebene Geschäftstätigkeit im Westen (zum Beispiel in Polen) brachte kaum noch etwas ein, als dieser Restbestand 1950 liquidiert wurde. Die beiden anderen russischen Firmen besitzen ebenfalls keine bedeutenden Nachfolger im Jahr 1995 und werden daher als schrumpfende Unternehmen ohne Marktwert behandelt. In allen Fällen, in denen westliche Unternehmen im Staatseigentum blieben, haben wir den Wert der Aktiva von 1995, korrigiert um etwaige Verbindlichkeiten, stellvertretend für den Marktwert benutzt. 


\section{Sind die Großunternehmen zwischen 1912 und 1995 größer oder kleiner geworden?}

Vier Fünftel der Großunternehmen, die auf unserer Liste des Jahres 1912 verzeichnet sind, stammen aus den wichtigsten Industrieländern dieser Zeit: aus Deutschland, Großbritannien und vor allem aus Amerika, auf das allein über die Hälfte dieser Firmen entfällt. Die meisten Unternehmen verfügten über ein erhebliches Qualifikationspotential, über Vorreitervorteile, steigende Skalenerträge, reichhaltige Erfahrungen oder über einen technologischen Vorsprung in einem solchen Maße, daß ihnen ein Konkurrenzvorteil gegenüber den anderen Firmen sicher war. Ein solches Übergewicht war in vielen Fällen das Ergebnis institutioneller Lernprozesse, die nicht beliebig wiederholt werden konnten, weil sie mit der speziellen Unternehmensgeschichte verbunden waren. Viele bekannte Musterbeispiele für die Lernfähigkeit von Institutionen, die in der Literatur ${ }^{1}$ abgehandelt werden, zeigen sich auch in der Gruppe der Großunternehmen von 1912. Die Firma Westinghouse Air Brake beispielsweise gewann Marktmacht über die Eisenbahngesellschaften, die zu ihren Kunden gehörten, indem sie Standards für das Schienennetz setzte. Andere Unternehmen wiederum, zu denen Shell, Jersey Standard (Exxon), Rio Tinto (RTZ) und Phelps Dodge gehörten, erwarben ihre Marktmacht dadurch, daß sie die Rohstoffe oder die Vertriebswege kontrollierten, oder - wie im Fall von Eastman Kodak und Siemens - durch die breite Einführung neuer Technologien. Wieder anderen Unternehmen wie Guiness, Procter \& Gamble und Lever Brothers gelang dies, indem sie den Weg für die Einführung von Markenartikeln auf den großen städtischen Märkten bahnten.

Verständlicherweise richtet die Literatur zur Unternehmensgeschichte ihre Aufmerksamkeit in erster Linie auf solche Firmen, wie sie oben aufgeführt wurden, weil deren Entwicklung von einem fortdauernden Erfolg begleitet war. Darin liegt jedoch die Gefahr, daß die Großunternehmen verzerrt wahrgenommen werden und als Zentren eines sich selbst reproduzierenden Leistungsvermögens erscheinen. Tatsächlich verleitet ihr langanhaltender Erfolg sehr leicht zu der Auffassung, daß diese Unternehmen ihre bereits erworbenen Marktpositionen und ihre organisatorischen Routinen nicht nur verteidigen, sondern durch die Anwendung dessen, was sie bereits gelernt haben, nämlich dynamische und lernfähige Organisationen aufzubauen, ihre Vorteile verstärken und so den Umkreis ihres Leistungspotentials ständig erweitern können. Die Mittel dazu liegen in der räumlichen, oft internationalen Ausdehnung des Geschäftsfeldes, in der Diversifikation der Pro-

1 Siehe zum Beispiel Usselman (1997) für Westinghouse Air Brake, Samber (1997) für Firmen in Pittsburgh, Genovese und Mullen (1997) für American Sugar, Lamoreaux und Sokoloff (1997) für Pullman. 
dukte oder in der Institutionalisierung von Innovationsprozessen in Abteilungen für Forschung und Entwicklung. Es ist sicherlich unbestreitbar, daß einige Großunternehmen wie Procter \& Gamble, Du Pont, Shell, Siemens und andere genau diesen Weg beschritten haben, aber es stellt sich die Frage, ob diese Entwicklung typisch für die Mehrzahl der Großunternehmen ist. Diese Frage wurde selten für eine Gruppe von Unternehmen untersucht, die am Anfang ihres Entwicklungsprozesses festgelegt wurde, um ihr weiteres Schicksal zu verfolgen, sondern öfter anhand allseits bekannter Unternehmen, die ihren Weg erfolgreich beschritten haben.

Wer erinnert sich jedoch heute noch an deutsche Großunternehmen wie Hohenlohe Eisen und Stahl, an britische wie Metropolitan Carriage und US-amerikanische wie Central Leather, die Nevada Consolidated and Utah Copper Group oder Cudahy Packing? Und wenn man sich an diejenigen Firmen erinnert, die einen drastischen Verfall erlitten haben, dann geschieht dies zumeist deshalb, weil sie ein Musterbeispiel für Organisationen abgeben, die bei der Entwicklung ihres Potentials versagt haben.

Die Zusammenstellung der Großunternehmen des Jahres 1912 - und die Untersuchung ihres Lebensweges - gestattet es uns, die weitere Frage zu beantworten, wie typisch die Entwicklungsverläufe derjenigen Firmen sind, an die wir uns erinnern, verglichen mit den anderen, die einfach in Vergessenheit geraten sind. Ist es für Unternehmen leicht, das eigene Leistungspotential zu entwickeln und Lerneffekte zu realisieren, oder ist dies schwierig zu bewerkstelligen? Die Tabelle 1 zeigt, daß etwas mehr als ein Viertel aller Firmen sowohl unabhängig blieb als auch Wachstumsprozesse durchlaufen hat, und etwas mehr als einem Fünftel gelang es, innerhalb der Gruppe der größten 100 Firmen zu verbleiben, wenn die Kapitalwerte von 1995 zugrunde gelegt werden. Dennoch waren diese günstigen Ergebnisse in der Gruppe der Großunternehmen weniger verbreitet als das Verschwinden der Aktiengesellschaften zum einen oder die Erfahrung eines - zumindest drohenden - Konkurses zum anderen. Zu diesen ungünstigen Ergebnissen zählen wir auch die Verstaatlichungen, sofern sie auf längere Sicht den Konkurs der Firmen verhinderten. Es ist hervorzuheben, daß annähernd drei Viertel aller Großunternehmen aus der Gruppe der 100 entweder ganz verschwunden oder geschrumpft sind, wenn man die Jahre 1912 und 1995 miteinander vergleicht. Zwar ist es richtig, daß die durchschnittliche Großunternehmung im Jahr 1995 (oder zu einem früheren Zeitpunkt) gröBer war als 1912, aber dieser Durchschnittswert wurde durch die wenigen Beispiele eines außergewöhnlich starken Unternehmenswachstums verzerrt; weniger als ein Drittel der Unternehmen zeigt überhaupt ein positives Wachstum, der Modalwert (der häufigste Wert) liegt bei Null. Insgesamt kann man sagen, daß bei dieser hochgradig schiefen Verteilung der Durchschnitt des Quotienten aus den Kapitalwerten der Jahre 1995 und 1912 je 
nach den getroffenen Annahmen zwischen 1,1 und 1,4 lag, wobei ein Wert um 3 notwendig war, um in der Liste der weltweit 100 größten Unternehmen zu verbleiben. ${ }^{2}$ Auffällig ist, daß der Niedergang unter den größten der 100 Großunternehmen besonders ausgeprägt war.

Um Marshalls Analogie zwischen den »Bäumen des Waldes« und den Unternehmen etwas zu erweitern, könnte man also nach den vorliegenden Befunden sagen, daß die »Giant Redwoods « unter ihnen - die Großunternehmen mithin - langfristig auch dem Niedergang und Verfall ausgesetzt sind, wobei es die ganz besonders großen Unternehmen häufiger als die übrigen trifft. Ökonomen sind nur zu gut dafür bekannt, daß sie sich selten festlegen, wenn sie von der langen Frist sprechen; in unserem Zusammenhang verstehen wir darunter, daß Großunternehmen eine ausgeprägte Neigung aufweisen, im Verlauf einiger Jahrzehnte zu verfallen. Um die Lebenserwartung der Großunternehmen genauer zu beschreiben, kann der Begiff der »Viertelwertszeit« eingeführt werden, womit wir die Zeitspanne bezeichnen, die verstreichen muß, bis ein Viertel aller Großunternehmen (einer gegebenen Population) durch Konkurs, durch Fusion oder Verstaatlichung verschwunden ist; in analogem Sinne wird der Begriff der »Halbwertszeit« benutzt. Unsere Untersuchung zeigt, daß die Viertelwertszeit der Großunternehmen des Jahres 1912 bei dreiunddreißig Jahren lag und daß sich diese Unternehmen gegenwärtig ihrer Halbwertzeit nähern. Von den 100 Großunternehmen aus dem Jahr 1912 bestehen noch 52 formal als selbständige Firmen. Sicherlich haben kleine Firmen im Durchschnitt eine kürzere Lebenserwartung als Großunternehmen ${ }^{4}$, aber sowohl die kleinen als

2 Um die 100. Firma von ihrem Rangplatz in der Liste der 100 größten Firmen des Jahres 1995 zu verdrängen, hätte die kleinste Firma auf der Liste von 1912 eine Leistungsrate von 4,0 erreichen müssen. Sehr viel weniger war natürlich für die größten Firmen von 1912 erforderlich: das größte Unternehmen des Jahres 1912, die Firma US Steel mußte auf ein Zehntel ihrer vormaligen Größe schrumpfen, um aus der Liste der 100 Größten von 1995 herauszufallen. Wenn sie ihre reale Größe beibehalten hätte, dann läge ihr Kapitalwert im Jahr 1995 bei 90,5 Mrd. Dollar und damit kurz hinter dem Unternehmen Royal Dutch Shell, das 1995 die Liste der Großunternehmen mit einem Wert von 107,6 Mrd. Dollar anführte. Der tatsächliche Wert der Firma USX (des Nachfolgers von US Steel) im Jahr 1995 lag jedoch bei 8,7 Mrd. Dollar, selbst nach der Fusion mit einem Großunternehmen aus der Ölindustrie; damit fiel USX aus der Gruppe der 100 größten Unternehmen heraus. Das durchschnittliche Großunternehmen des Jahres 1912 hätte eine Leistungsrate von 2,7 erreichen müssen, um ein durchschnittliches Großunternehmen von 1995 zu werden; der Kapitalwert hätte von 81 Mill. Dollar auf 218 Mill. Dollar steigen müssen, gemessen in den Aktienpreise des Jahres 1912.

3 Dies wird durch den Abstand deutlich, der zwischen der ungewichteten durchschnittlichen Leistungsrate $(1,4)$ und der geringeren, mit der Größe von 1912 gewichteten Durchschnittsrate $(1,2)$ besteht; siehe Tabelle 1 .

4 Die meisten Studien über neugegründete, kleine Firmen kommen auf eine Halbwertszeit, die im niedrigen einstelligen Bereich liegt. Zu der Zeit, als Marshall seine Untersuchungen begann, hatten die englischen Aktiengesellschaften eine Halbwertszeit von rund sieben Jahren (Shannon 1933). 
auch die großen Firmen werden absterben. Sie unterscheiden sich in dieser Hinsicht nur durch ihre Lebenserwartung, aber selbst hier ist der Unterschied erstaunlich gering: um die Halbwertszeit einer Aktiengesellschaft um ein Jahr zu verlängern, ist es notwendig, ihre Größe um das Dreiundzwanzigfache zu steigern.

Tabelle 1: Zusammengefaßte Darstellung der langfristigen Leistung der 100 größten Unternehmen des Jahres 1912 im Jahr 1995

\begin{tabular}{|l|c|}
\hline & $\begin{array}{c}\text { Wahrschein- } \\
\text { lichkeit }\end{array}$ \\
\hline Fortbestehende Unternehmen & $21 \%$ \\
\hline - in der Gruppe der 100 größten Unternehmen & $28 \%$ \\
\hline - in 1995 größer als in 1912 & $52 \%$ \\
\hline - eindeutig unabhängiges Unternehmen, gleichgültig von welcher Größe \\
\hline $\begin{array}{l}\text { Firmen, die aufgelöst oder verstaatlicht wurden oder unter } \\
\text { Schutzmaßnahmen gegen Konkurs standen }\end{array}$ & $29 \%$ \\
\hline $\begin{array}{l}\text { Leistungsrate (Quotient der Aktienwerte 1995/1912 wie oben definiert), } \\
\text { ein Wert von 1 indiziert Stabilität, kleiner als 1 Niedergang }\end{array}$ \\
\hline $\begin{array}{l}\text { - Durchschnitt der fortbestehenden Unternehmen } \\
\text { - Durchschnitt der erloschenen Unternehmen } \\
\text { - (letzter verfügbarer Wert / Wert von 1912) }\end{array}$ \\
\hline - der gesamte ungewichtete Durchschnitt & 2,0 \\
\hline - der gesamte Durchschnitt, gewichtet mit den Kapitalwerten von 1912 & 0,6 \\
\hline $\begin{array}{l}\text { - der gesamte ungewichtete Durchschnitt, in dem alle erloschenen } \\
\text { Unternehmen gleich Null gesetzt werden }\end{array}$ & 1,4 \\
\hline - der gesamte gewichtete Durchschnitt ohne Doppelzählungen * & 1,2 \\
\hline $\begin{array}{l}\text { - Anteil der gewachsenen Unternehmen an den 100 Großunternehmen } \\
\text { (d.h. Leistungsrate größer als eins) }\end{array}$ & $35 \%$ \\
\hline - Modalwert (d.h. häufigste anzutreffende Leistungsrate) & 1,1 \\
\hline
\end{tabular}

*) Firmen werden Null gesetzt, wenn sie von einem anderen Unternehmen aus der Liste der 100 dauerhaft erworben wurden.

Quelle: Berechnungen des Autors (ausführliche Angaben in Schmitz 1995 und Hannah 1997)

Das kleinste Unternehmen aus der Liste von 1912 hatte (in Aktienpreisen von 1995 gerechnet) einen Marktwert von drei Mrd. Dollar, das größte kam auf 90 Mrd. Dollar; durchschnittlich gesehen konnten diese Firmen ihren Wert jedoch kaum erhöhen, bei vielen wurde er vermindert, bei einigen ist er vollständig verschwunden. Dieser Befund zeigt zudem, daß die behaupteten außergewöhnlich starken Umschichtungen in der Unternehmensrangfolge, die sich aus dem Aufstieg starker und dem Abstieg schwacher Firmen ergeben, kein modernes Phänomen sind, das mit der Liberalisierung des Welthandels entstanden ist, sondern bereits in früheren Perioden beo- 
bachtet werden konnte, die durch wirtschaftliche Autarkiebestrebungen der einzelnen Staaten und eine ökonomische Stagnation gekennzeichnet waren. Der Verfall von Großunternehmen ist allgegenwärtig in einer sich ständig wandelnden Welt, und in dem folgenden Witz steckt viel Wahrheit. Frage: Wie baue ich ein erfolgreiches Kleinunternehmen auf? Antwort: Kaufe ein großes und warte!

Eine Erklärung für die stark ausgeprägte Tendenz der Großunternehmen zur Stagnation und zum Niedergang wurde insbesondere von Stigler (1965) aufgrund seiner Untersuchung der Firma US Steel in der Strategie von marktbeherrschenden Unternehmen gesehen, lediglich die Gewinne der Anteilseigner zu maximieren. Für solche dominierenden Firmen könne es sinnvoller sein, ihre Monopolposition preislich auszunutzen und dabei Marktanteile an neu hinzutretende Konkurrenten zu verlieren, als eine Preispolitik zu verfolgen, die den Marktzugang neuer Unternehmen verhindert. Diese Strategie wäre insbesondere dann wahrscheinlich, wenn es darum ginge, bei den Kartellbehörden keinen Verdacht zu erregen. Die Betrachtung der Unternehmensgeschichten von einigen verfallenden Firmen aus unserer Liste zeigt, daß von einem derart geplanten Niedergang nicht gesprochen werden kann, sondern letzterer das Ergebnis von kopflosen Reaktionen der Unternehmensleitung gewesen ist. Wir haben die durchschnittlichen Erträge der Aktienbesitzer nicht untersucht, aber unser allgemeiner Eindruck, der aus der Betrachtung der Firmengeschichten gewonnen wurde, legt nahe, daß die Zahlung von verminderten Gewinnen an die Anteilseigner in der Phase des Niedergangs vom Management dazu benutzt wurde, die schwindende Leistungsfähigkeit der Unternehmen zu übertünchen, aber nicht als eine besondere Großzügigkeit gegenüber den Aktionären in einer Phase sinkender Marktanteile gewertet werden kann. Man kann die Hypothese von Stigler sicher weiter untersuchen, aber es scheint, daß man damit nur zu einem kleinen Teil die schrumpfende Unternehmensgröße erklären kann.

Für die Industrieökonomen ist die Bedeutung dieser Beobachtungen klar: Während wir unsere Aufmerksamkeit wie selbstverständlich auf die erfolgreichen Unternehmen richten, die gelernt haben, ihr Leistungspotential zu vergrößern und zu sichern, ist dies doch ein Ergebnis, das die riesigen Unternehmensbürokratien in der Regel nicht ohne weiteres zuwege bringen. Tatsächlich ist es so, daß die dem 20. Jahrhundert zugeschriebene angeblich auf allen nationalen Märkten wirksame Tendenz zu einer stärkeren Konzentration in der Industrie weder allgemein verbreitet noch nachhaltig wirksam ist (Hannah 1995), und möglicherweise ist sie auch weltweit nur sehr schwach ausgeprägt.

Man sollte daher für die Ursachen, die der Zersetzung unternehmerischer Leistungspotentiale zugrundeliegen, die gleiche Aufmerksamkeit entwi- 
ckeln wie für die Faktoren, die den Aufbau solcher Potentiale befördern. Aktiengesellschaften können vergessen und lernen; ihre ererbten Lernerfolge können in einem neuen sozialen und ökonomischen Umfeld überflüssig oder sogar dysfunktional werden; Vorreitervorteile können sich als flüchtig erweisen; Fähigkeiten, von denen man annahm, daß nur das eigene Unternehmen über sie verfüge, können von konkurrierenden Firmen nachgebildet oder verbessert werden (vgl. dazu Raff/Temin 1997, Hounshell 1997, Henderson/Clark 1990). Solche Resultate treten doppelt so häufig auf wie eine erfolgreiche Erweiterung des Leistungspotentials, über das Großunternehmen verfügen.

Könnten wir vorab erkennen, was den Erfolg eines Unternehmens bestimmte, so könnten wir das Blatt der ökonomischen Evolution wenden und sehr reiche Männer werden. Man wird indes nicht überrascht sein zu erfahren, daß dies ein sehr schwieriges Unterfangen ist und daß alle diejenigen, die den Versuch bislang unternommen und solche Rezepte erarbeitet haben, nicht sonderlich überzeugen konnten. Obgleich die Anzahl der Großunternehmen auf unserer Liste zu gering ist, um statistisch signifikante Ergebnisse zu erhalten, so gestattet sie es uns aber zumindest, einige von diesen Rezepturen für den Unternehmenserfolg in Frage zu stellen, selbst wenn sie aus der Rückschau, d.h. aus erfolgreichen, aber vergangenen Unternehmensentwicklungen, gewonnen wurden. Das können wir auch etwas anders formulieren: Wenn die ex post $»$ Vorhersage schon nicht einfach ist, so können wir sicher sein, daß die ex ante Vorhersage einige Probleme aufwerfen wird. Wir sollten mithin diejenigen Berater für strategische Unternehmensentwicklung am höchsten schätzen, die am bescheidensten auftreten.

\section{Nationale Unterschiede im Erfolg der Unternehmen}

Chandler hat in seiner kürzlich erschienenen, international vergeichenden Studie ein inzwischen weit verbreitetes Modell entwickelt, welches den Niedergang von Aktiengesellschaften erklären soll; darin wird der wirtschaftliche Abstieg, den Großbritannien im Verhältnis zu Deutschland und Amerika im Verlaufe des 20. Jahrhunderts erfahren mußte, mit dem Versagen der Großunternehmen begründet, große und effiziente Verwaltungen in den Aktiengesellschaften einzuführen (Chandler 1990). Diese These wird aus einer überzeugenden Erörterung der unternehmerischen Leistungspotentiale entwickelt, aber die darin enthaltenen internationalen Vergleiche sind von mehreren Seiten her angreifbar (Alford 1994, Hannah 1995). Unsere Gruppe von ausgewählten Großunternehmen kann sicher nicht herangezogen werden, um die These zu unterstützen, wonach die britischen Großunternehmen ihre Leistungsfähigkeit nicht so gut erhalten konnten wie 
die deutschen oder amerikanischen. Wie die Tabelle 2 zeigt, trifft genau das Umgekehrte zu: die britischen Firmen überlebten häufiger, blieben mit höherer Wahrscheinlichkeit in der Gruppe der hundert größten Unternehmen und erreichten im Durchschnitt eine bessere Entwicklung ihrer Leistungsfähigkeit als die amerikanischen und die deutschen Unternehmen.

Tabelle 2: Die Unterschiede in der Leistung der Großunternehmen von 1912 nach Nationen betrachtet

\begin{tabular}{|c|c|c|c|c|}
\hline Land & USA & Deutschland & UK & andere \\
\hline Anzahl der Firmen mit Hauptsitz (1912) & 54 & 14 & $15^{*}$ & $17 *$ \\
\hline $\begin{array}{l}\text { Durchschnittlicher Aktienwert im Jahr } 1912 \\
\text { in Millionen Dollar }\end{array}$ & 90 & 59 & 95 & 56 \\
\hline \multicolumn{5}{|l|}{ Überlebenschance } \\
\hline noch in der Liste der Besten 100 in 1995 & $19 \%$ & $29 \%$ & $47 \%$ & $0 \%$ \\
\hline $\begin{array}{l}\text { als unabhängiges Unternehmen von belie- } \\
\text { biger Größe überlebt }\end{array}$ & $48 \%$ & $57 \%$ & $60 \%$ & $53 \%$ \\
\hline \multicolumn{5}{|l|}{ Verbesserung des Leistungspotentials } \\
\hline $\begin{array}{l}\text { Anteil der Unternehmen, die gewachsen } \\
\text { sind (Leistungsrate ist größer als 1) }\end{array}$ & $26 \%$ & $43 \%$ & $40 \%$ & $18 \%$ \\
\hline $\begin{array}{l}\text { ungewichtete durchschnittliche Leistungs- } \\
\text { rate der verbliebenen Großunternehmen }\end{array}$ & 2,3 & 1,9 & 2,7 & 0,7 \\
\hline $\begin{array}{l}\text { ungewichtete durchschnittliche Leistungs- } \\
\text { rate der aus der Liste verschwundenen } \\
\text { Großunternehmen }\end{array}$ & 0,7 & 0,1 & 0,7 & 0,3 \\
\hline ungewichtete durchschnittliche Gesamtrate & 1,5 & 1,2 & 1,9 & 0,5 \\
\hline Variationskoeffizient & $(135)$ & $(104)$ & (123) & (164) \\
\hline $\begin{array}{l}\text { Verhältnis des realen BSP von } 1995 \text { zum } \\
\text { realem BSP von } 1912\end{array}$ & 8 & 9 & 4 & --- \\
\hline
\end{tabular}

* Royal Dutch Shell wird vollständig als britische Firma ausgewiesen. Quelle: eigene Berechnungen des Autors.

Diese Zahlen laden zu einigen Spekulationen über die Bedeutung Großbritanniens als Standort für die Großunternehmen ein, vor allem wenn man die deutlich geringeren Wachtumsraten der britischen Ökonomie in den Jahrzehnten nach 1912 betrachtet. Möglicherweise zeigten die britischen Großunternehmen ein größeres Stehvermögen, weil sie im Jahr 1912 ihren Sitz in einem Land hatten, das den Handel schon liberalisiert hatte, während die amerikanischen Unternehmen über die Maßen durch hohe Zollschranken und die deutschen durch gemäßigte Zölle geschützt waren (Capie 1994, 59). Somit war die Leistungsfähigkeit der britischen Unterneh-

5 Die Verwendung des US-amerikanischen Aktienmarktindexes als Deflator anstelle des britischen verzerrt die Ergebnisse zuungunsten der Firmen mit Sitz in Großbritannien. 
men schon 1912 deutlich härteren, nämlich globalen Marktbedingungen unterworfen. Zudem tendierten die britischen Großunternehmen bereits in dieser Zeit dazu, einen ungewöhnlich hohen Anteil ihrer Ressourcen weltweit einzusetzen. Darüber hinaus waren sie durch den inländischen Markt weniger in ihrer Entwicklung eingeschränkt und bereits in höherem Maße auf den globalen Wettbewerb eingestellt als die deutschen und amerikanischen Großunternehmen, weil ungefähr ein Drittel ihrer Geschäftstätigkeit in Übersee stattfand; das war wahrscheinlich doppelt so viel wie in einem durchschnittlichen amerikanischen oder deutschen Großunternehmen in jener Zeit (Hannah 1997).

Es wäre aber ein Fehler, solche Spekulationen allzu weit zu treiben. Unsere Ergebnisse reichen jedoch aus, um die Vorstellung in Zweifel zu ziehen, $\mathrm{da} ß$ es außergewöhnlich wenige britische Großunternehmen gab oder daß letztere besonders anfällig für eine mangelnde Entwicklung ihrer Leistungspotentiale waren. Sie verweisen auch darauf, daß Chandlers Schlußfolgerungen für die Bedeutung der nationalen Differenzen wahrscheinlich nicht bestätigt werden können, wenn man eine größere Anzahl von mittleren Industrieunternehmen untersucht. ${ }^{6}$ Broadberry (1998) hat gezeigt, daß Amerika und Deutschland im zwanzigsten Jahrhundert Großbritannien nicht deshalb überholt haben, weil sie ihre Arbeitsproduktivität in der Industrie im Vergleich zu Großbritannien gesteigert haben, sondern zum einen aufgrund einer Verlagerung von Ressourcen aus der Landwirtschaft in die Industrie - eine Option, die dem industrialisierten Großbritannien nicht mehr offenstand - und zum anderen dadurch, daß die außergewöhnlich hohe Produktivität des britischen Dienstleistungsbereichs übertroffen wurde. Ein Beispiel für die USA stellen Raff und Temin (1997) dar und Wright (1997) liefert eine allgemeine Behandlung dieses Themas. Broadberrys sektorale Disaggregation der Produktivitätsentwicklung gibt somit einen weiteren Beleg dafür, wie fragwürdig jene Position ist, derzufolge die Unterschiede in der nationalen Konkurrenzfähigkeit im 20. Jahrhundert wesentlich durch die Produktivitätsdifferenzen in den Industrieunternehmen hervorgebracht wurden. Auf einer etwas allgemeineren Ebene hat Robert Reich einige Fehler in der Auffassung herausgearbeitet, daß die leistungsfähigsten Unternehmen und die Ökonomien ganzer Nationalstaaten eng miteinander verbunden wären (Reich 1992, vgl. auch Lazonick 1993). Außerdem kann man die Möglichkeit nicht ausschließen, daß eine lebhafte Konkurrenz der kleineren Firmen, die auch Großunternehmen verdrängen können (selbst solche mit Vorreitervorteilen), das wesentliche Kennzeichen

6 Teece $(1993,214)$ hebt hervor, aus Chandlers eigenen Daten über die größten 200 Unternehmen jedes Landes gehe hervor, daß die führenden deutschen Unternehmen eine geringe Wahrscheinlichkeit aufwiesen, ihre Position zu halten, was er mit den Kriegsfolgen in Deutschland erklärte (vgl. dazu auch Cassis 1997, Broadberry 1998, Mishina 1997). 
für eine starke Ökonomie ist und daß sich an diesem Punkt Amerika und Deutschland auf der einen Seite von Großbritannien auf der anderen stärker unterscheiden als durch ihre Großunternehmen.

Tabelle 3: Die räumliche Verteilung der 100 größten Unternehmen (gemessen am Aktienwert) weltweit

\begin{tabular}{|lcc|}
\hline Land, in dem sich der Hauptsitz & 1912 & 1995 \\
befindet & & 40 \\
\hline USA & 54 & 12,5 \\
Großbritannien & 14,5 & 7 \\
Deutschland & 14 & 5 \\
Frankreich & 6 & 1 \\
Südafrika & 4 & 0 \\
Rußland & 3 & 0 \\
Belgien & 2 & 0 \\
Mexiko & 1 & 0 \\
Luxemburg & 1 & 2,5 \\
Niederlande & 0,5 & 21 \\
Japan & 0 & 4,5 \\
Schweiz & 0 & 2,5 \\
Schweden & 0 & 1 \\
Australien & 0 & 1 \\
Italien & 0 & 1 \\
Finnland & 0 & 1 \\
Korea & 0 & 100 \\
Insgesamt & 100 & \\
\hline
\end{tabular}

Quellen: für 1912 Berechnungen des Verfassers; für 1995 berechnet nach den Angaben von Business Week (31.5.1996), nur für die Industrieunternehmen.

Wenn die Unternehmenshistoriker der Bedeutung nationaler Unterschiede für die Erfolge der Großunternehmen schon so viel Aufmerksamkeit widmen, dann sollten sie zumindest klarere Hypothesen formulieren, die den Tatsachen besser entsprechen. Bis es soweit ist, gehen wir am besten von der Annahme aus, daß die Nationalität keine bedeutsame Variable ist, um den Erfolg der großen Unternehmen zu erklären, soweit es sich um die entwickelten Industrienationen handelt. Die Methoden der erfolgreichen Großunternehmen und Nationalstaaten ${ }^{7}$ lassen sich ebenso leicht wiederholen und finden eine ebensolche Verbreitung wie - so muß man wohl sinnvollerweise sagen - die Fehler der erfolglosen.

Wie die Daten der Tabelle 3 zeigen, beherbergen heute mehr Länder industrielle Großunternehmen als im Jahr 1912, wobei diese Zunahme eher zu

7 Die Belege, die in der Arbeit von Kim (1995) enthalten sind und sich auf die Verbreitung der industriellen Leistungsfähigkeit in den Regionen der USA seit 1930 beziehen, stützen unsere Vermutung. 
Lasten der USA und Deutschlands gegangen ist als zu Lasten Großbritanniens. ${ }^{8}$ Um die erfolgreichen von den weniger erfolgreichen Nationalökonomien zu unterscheiden, muß man sich offenbar andere Kriterien suchen, die Erfolge der Großunternehmen lassen sich zu diesem Zweck nicht heranziehen.

\section{Gibt es aufsteigende und absteigende Industriezweige oder aufsteigende und absteigende Firmen?}

Auf den ersten Blick scheinen die Industriezweige, in denen im Jahr 1912 die Großunternehmen konzentriert waren, eine konsistentere Entwicklung ihrer Leistung zu zeigen als die jeweiligen Nationalökonomien. Wie die Tabelle 4 zeigt, war rund die Hälfte aller Firmen in fünf Branchen zu finden: Kohlebergbau, Textil- und Lederverarbeitung, Nichteisenmetalle und sonstiger Bergbau, Schwerindustrie und schließlich der Maschinenbau. In diesen Industrien schrumpfte die durchschnittliche Firma bis zum Ende des Untersuchungszeitraums auf weniger als die Häfte ihrer Größe, die sie im Jahre 1912 erreicht hatte, und nur eine sehr geringe Zahl von Großunternehmen konnte weiter wachsen. Die Erfolgsgeschichten in diesen »alten« Industrien kamen auf ganz verschiedenen Wegen zustande: oft wurde die Firma verkauft, zum Beispiel Metropolitan Carriage, die Ausrüstungsgüter für die Eisenbahn herstellte und im Jahr 1919 eine Leistungsrate von 2,0 erreichen konnte; andere Unternehmen waren sowohl in ihrem alten Kerngeschäft als auch auf neuen Geschäftsfeldern erfolgreich, wie das Beispiel der Firma Mannesmann zeigt, die mit einer Rate von 2,7 im Jahr 1995 den besten Wert in dieser Gruppen von Industriezweigen erreichen konnte. Von den erfolgreichen Unternehmen haben nur wenige den Industriezweig vollständig gewechselt: so verlagerte American Can sein Tätigkeitsfeld auf die Finanzdienstleistungen (mit einer Leistungsrate von 1,9) und der französische Stahlgigant Schneider verlegte sich auf die Elektrotechnik (mit einer Leistungsrate von 1, die Stabilität anzeigt). Solche »neuen« Industriezweige gewährten zweifelsohne bessere Wachstumschancen, aber viele der »alten « Industrien des Jahres 1912 hatten keine besonderen Erfahrungen, die sie in die neuen Branchen transferieren konnten, um damit den Einschränkungen in ihren alten Märkten zu entgehen. Wie die Untersuchung der Großunternehmen im Kohlebergbau, der Textilindustrie und in der Zulieferindustrie für die Eisenbahnen zeigt, entstanden aus der Geschwindigkeit,

8 Die Zahl der Fälle ist gering und man sollte nicht versuchen, zuviel aus ihnen herauszulesen. Vergleicht man die Zahlen für 1995 mit denen aus der Tabelle 2 für die überlebenden Großunternehmen des Jahres 1912, dann zeigt sich, daß in den USA zahlreiche neue Großunternehmen entstanden sind, während die Entwicklung der alten Großunternehmen von 1912 dort nicht sonderlich erfolgreich verlaufen ist. In Deutschland hingegen ist dies nicht eingetreten: Seine industrielle Stärke liegt im Mittelstand. 
mit der individuelle Märkte zusammenbrachen, und aus den Grenzen, die dem Transfer unternehmerischer Leistungspotentiale von einem Industriezweig in den anderen im Jahr 1912 gegenüberstanden, besonders große Hindernisse für einen solchen Wechsel des Geschäftsfeldes. Für solche Firmen könnte die aussichtsreichste Lösung in einer Übernahme durch andere Unternehmen gelegen haben, um den Wert der wenigen verbliebenen Fähigkeiten zu maximieren, die auf andere Industriezweige übertragbar waren. Aus dem gleichen Grund hatten es - vielleicht - diejenigen Firmen, die bereits 1912 in den schnell wachsenden Wirtschaftszweigen wie der Ölindustrie, der Chemie oder der Elektrotechnik produzierten, etwas leichter, ihren Anfangsbestand an Erfahrungen, Qualifikationen und Fähigkeiten zu verwerten und ein dynamisches Leistungspotential aufzubauen. Die Großunternehmen in diesen Industrien konnten im Durchschnitt ihre reale Größe immerhin mehr als verdoppeln. Auch solche Firmen, die Markenartikel herstellten, neigten eher zum Wachstum als zum Niedergang; in diesem Sektor gab es 1912 die meisten Großunternehmen neben der Schwerindustrie.

Tabelle 4: Leistungsunterschiede zwischen den Großunternehmen des Jahres 1912 nach Industriezweigen

\begin{tabular}{|l|c|l|l|l|l|l|}
\hline Industriezweige & A & B & C & D & E & F \\
\hline Textilien und Leder & 4 & 0,4 & 0,2 & 0,1 & 0,1 & 79 \\
\hline Kohlebergbau & 7 & 0,4 & 0,1 & 0,2 & 0,3 & 185 \\
\hline Maschinenbau & 10 & 0,9 & 0,2 & 0,4 & 0,6 & 165 \\
\hline Nichteisenmetalle etc. & 14 & 1,2 & 0,4 & 0,4 & 0,5 & 119 \\
\hline Schwerindustrie & 18 & $1,5 ?$ & $0,1 ?$ & $0,6 ?$ & $0,7 ?$ & $175 ?$ \\
\hline Markenartikel & 18 & 1,6 & 1,1 & 1,3 & 1,9 & 142 \\
\hline Chemie & 10 & 0,4 & 2,7 & 2,4 & 1,9 & 79 \\
\hline Elektroindustrie & 5 & 0,4 & $3,0 ?$ & 2,7 & 1,9 & 70 \\
\hline Ölindustrie & 14 & 1,2 & $3,2 ?$ & 3,7 & 2,3 & 62 \\
\hline $\begin{array}{l}\text { alle Großunternehmen } \\
\text { des Jahres 1912 }\end{array}$ & 100 & 8,1 & $1,1 *$ & 1,4 & 1,9 & 140 \\
\hline
\end{tabular}

A: Anzahl der Großunternehmen, die 1912 in dieser Industrie sind. B: Aggregierte Aktienwert aller Großunternehmen dieses Industriezweiges im Jahr 1912 in Mrd. Dollar. C: Der gesamte Erfolg eines Industriezweiges.** D: Der durchschnittliche Erfolg eines Industriezweiges.*** E: Standardabweichung. F: Variationskoeffizient.

* Siehe Tabelle 1 für die Bereinigung um Doppelzählungen.

** Durchschnittliche Leistungsrate gewichtet mit der Unternehmensgröße im Jahr 1912.

*** Ungewichtete durchschnittliche Leistungsrate. Wenn dieser Durchschnitt kleiner als der gewichtete Durchschnitt ist, dann zeigen die echten Riesenunternehmen bessere Erfolge als Unternehmen, die einfach nur groß sind.

Quelle: Eigene Berechnungen des Verfassers. 
Es wäre jedoch falsch anzunehmen, daß der Erfolg der Großunternehmen durch die Wahl eines aufsteigenden oder absteigenden Industriezweigs gleichsam vorherbestimmt wäre. Tatsächlich waren die Unterschiede innerhalb der Industriezweige größer als diejenigen zwischen ihnen. ${ }^{9}$ Selbst in einem so aussichtsreichen Wirtschaftszweig wie der Elektroindustrie, wo vier von sechs Firmen eine Leistungsrate größer als 1 erzielten, erreichten Westinghouse $(0,7)$ und AEG $(0,3)$ nur schwache Ergebnisse. Im großen und ganzen konnten sich die Ölunternehmen gut entwickeln, aber die Nachzügler konnten unter ihrem Wert von 1912 liegen, wenn sie verstaatlicht oder von anderen Firmen übernommen wurden. Weitere Beispiele dafür lassen sich leicht finden. Wie die letzte Spalte der Tabelle 4 erkennen läßt, zeigten sowohl die alten wie auch die neuen Industriezweige große Unterschiede in den Wachstumsraten: der Variationskoeffizient übersteigt den Wert 60 in allen Industrien und 120 in der Hälfte der Industriezweige. Dieses Muster der Entwicklung innerhalb der Wirtschaftszweige stimmt mit den Ergebnissen überein, zu denen andere Studien mit einer größeren Stichprobe von Unternehmen für kürzere Zeitspannen gekommen sind (Schmalensee 1985, Rumelt 1991). Dies zeigt, daß dynamische Wirtschaftssysteme zwar aus aufsteigenden und absteigenden Branchen bestehen, aber Unternehmen können in jedem Branchentyp ihre Konkurrenzvorteile entwickeln und behalten. Simplifizierende Rezepte für das Portfoliomanagement von Industrieunternehmen mögen den Unternehmensberatern ein Vermögen eingebracht haben, aber ein besserer Weg zum nachhaltigen Erfolg besteht darin, irgendwo entschieden und profitabel zu handeln, anstatt viel Geld für modische Unternehmensübernahmen mit dem Ziel der Diversifikation zu zahlen, wenn in diesen Industrien kein erkennbarer neuer Wert für die Eigentümer hinzukommt.

Somit stellt sich die Frage, wie die Großunternehmen ihre Position halten, ihren Kapitalwert erhöhen und ihr Leistungsvermögen erweitern können? Die einzige vernünftige Antwort lautet: Mit großen Schwierigkeiten! Es wäre sicherlich von großem Vorteil, wenn wir über Untersuchungen verfügten, aus denen hervorginge, welche Maßnahmen getroffen werden müßten, um den dauerhaften Erfolg der Großunternehmen sicherzustellen, aber ebendies entzieht sich unserem Verständnis.

Dieser Zustand ist keineswegs nebensächlich: Er bildet einen immanenten Bestandteil der Marktprozesse, die den Erfolg des Kapitalismus im 20.

9 Der Variationskoeffizient der Durchschnittsraten aller neun Industrien beträgt 95 . Somit ist die Variation innerhalb der Mehrzahl der Wirtschaftszweige (Kohle, Maschinenbau, Nichteisemmetalle etc., Eisen und Stahl und Markenartikel) größer als diejenige zwischen den Industriezweigen, und in den vier Sektoren mit der geringsten Varianz (Textilien, Chemie, Elektrotechnik und Öl) ist die Variabilität beinahe immer noch so groß wie zwischen den Sektoren. 
Jahrhundert tragen. Die meisten Unternehmen, die wir untersucht haben, hatten 1912 einen außerordentlichen Erfolg und ihre hohe Bewertung am Aktienmarkt folgte aus ihrer Fähigkeit, überdurchschnittlich hohe Profite zu verdienen. Diese Profite waren in der Regel die Belohnung für große Investitionen in der Produktion, im Management und im Marketing, wie sie von Chandler (1990) beschrieben werden. Häufig kam noch mehr dazu, damit sich die Marktposition der Vorreiter im Sinne Chandlers festigen konnte, nämlich ein technologischer Vorsprung, die exklusive Verfügung über Rohstoffe, ein bedeutendes Vertriebssystem oder ein bekannter Markenname. Jedoch sind solche Vorteile häufig nur zeitweilig vorhanden und unsicher: Patente erwiesen sich als begrenzt wirkungsvoll, Werbekampagnen ließen konkurrierende Markenartikel entstehen, neue Minen und Ölfelder wurden entdeckt, Techniken des Managements, der Produktion und des Marketings wurden von anderen Firmen kopiert. Selbst dort, wo dies nur schwer möglich war, konnte der anfängliche Vorsprung durch exogene Änderungen in der Produktionstechnik oder in den Eigenschaften der Märkte zunichte gemacht werden. Gefestigte Marktpositionen konnten ebenfalls durch die Kartellbehörden oder durch eine Enteignung gefährdet werden.

Waren solche Gefahren und Herausforderungen nicht vorhanden oder konnte die Firma ihnen mit einer eigenen Strategie erfolgreich begegnen, dann überlebte das Großunternehmen, aber unter dem fortwährenden Druck der Marktkräfte kann es nicht überraschen, daß der Niedergang und der vollständige Verfall bei den Großunternehmen dreimal so häufig zu beobachten war wie ein Unternehmenswachstum. Durch das Kopieren vorteilhafter Verfahren wurden die Profite der vormals führenden Unternehmen auf das normale Niveau herabgedrückt, und gleichzeitig konnte auf diese Weise der Nutzen aus den besseren Verfahren verallgemeinert werden. Eine überzeugende Erklärung für das Fortbestehen von Großunternehmen liegt in der gleichsam spezifischen Architektur, die es nur ihnen gestattete, beständig ihre frühen Erfolge zu wiederholen (Kay 1993). Solche unternehmerischen Architekturen müssen komplex und schwierig zu identifizieren und zu kopieren sein, weil andernfalls der zusätzliche Wert, den sie einem Unternehmen verschaffen, in der Konkurrenz herabgesetzt und verschwinden würde. Definitionsgemäß wissen wir nicht, wie diese Unternehmensarchitekturen beschaffen sind, aber es ist naheliegend, daß dieses Wissen in einem stillschweigenden Lernprozeß erworben und von einer Generation von Managern auf die andere übertragen wurde.

Sowohl der Antrieb zur Entwicklung von Konkurrenzvorteilen als auch der Antrieb, den erfolgreichen Unternehmen nachzueifern, waren in den Gesellschaften stark ausgeprägt, in denen die Großunternehmen 1912 tätig waren. Letztere verstanden es immer besser, die wirtschaftlichen Möglich- 
keiten zu übersehen und die gewinnbringenden Anlagen zu begutachten, wobei sie sich zunehmend wechselseitig Konkurrenz machten und sich gegenseitig zur weiteren Entwicklung ihrer Leistungspotentiale zwangen. In diesem Sinne ist die im Durchschnitt geringe Fähigkeit der Großunternehmen, ihr spezifisches Leistungsvermögen weiter zu entwickeln, das einmal ihre Größe begründet hatte, keineswegs als ein Anzeichen für die individuelle Schwäche einzelner Unternehmen zu deuten, sondern sie verweist auf ihre kollektive Stärke innerhalb des kapitalistischen Marktsystems. Marx verstand die »Widersprüche« dieses dialektischen Systems sehr genau. Paradoxerweise ist es so, daß die kapitalistischen Unternehmen durch das Streben nach Extraprofiten zu weiterem Wachstum veranlaßt werden, daß aber andererseits die Konkurrenz zwischen ihnen alle Gründe beseitigt, aus denen solche Extraprofite entstehen, so daß letztere nur als ein zeitweiliges Phänomen auftreten können. Marx beging jedoch den Fehler, dies als eine Schwäche jener Phase der ökonomischen Evolution zu betrachten, die er zu seiner Zeit beobachtete. Tatsächlich besteht die grundlegende Stärke des Kapitalismus als eines Systems der gesellschaftlichen Organisation in dieser Mischung aus Antrieben und Hindernissen und die ist nun dabei, sich weltweit auszudehnen.

Aus dem Englischen übersetzt von Klaus Schabacker

\section{Literatur}

Alford, W. E. (1994): Chandlerism, the New Orthodoxy of US and European Corporate Development, Journal of European Economic History, vol. 23, no.3, pp.631-643.

Broadberry, S. N. (1998): How did the United States and Germany become richer than Britain? A sectoral analysis and comparative productivity levels, 1870-1990, prepared for Leuven preconference, Nov. 1996, to Seville Int. Economic History Congress, Aug. 1998.

Capie, Forrest (1994): Tariffs and Growth, Some insights from the World Economy, 18501940, Manchester University Press, Manchester.

Cassis, Youssef (1997): Big Business: The European Experience, Oxford University Press, Oxford.

Chandler, Alfred D. (1990): Scale and Scope: The Dynamics of Industrial Capitalism, Belknap Press of HUP, Cambridge, Mass.

Genovese, David; Mullin, Wallace (1997): The Sugar Institute Learning to Control its Market, paper presented to the NBER Conference, Cam., Mass.

Hannah, Leslie (1974): Mergers in British Manufacturing Industry, 1880-1918, Oxford Economic Papers, 26.

Hannah, Leslie (ed.) (1976): Management Strategy and Business Development, Macmillan, London.

Hannah, Leslie (1995): The American Miracle, 1875-1950, and After: A View in the European Mirror, Business and Economic History, 24, 2, pp.197-263.

Hannah, Leslie (1996): Multinationality: Size of Firm Size of Country and History Dependence, Business and Economic History, 25.

Henderson, Rebecca M.; Clark, Kim B. (1990): Architectural innovation: the configuration of existing product technologies and the failure of established firms, Administrative Science Quarterly, vol. 35, 201, March, pp.9-30.

Hounshell, David A. (1997): Ford Automates, paper presented to the NBER Conference.

Kim, Sukkoo (1995): Expansion of markets and the geographic distribution of economic acti- 
vities: the trends in US regional manufacturing structure, 1860-1987, Quarterly Journal of Economics, 110, pp.881-908.

Jürgen Kocka (1976), 'Entrepreneurs and Managers in German Industrialisation', in: Mathias and Postan (1978).

Kogut, Bruce (ed.) (1993): Country Competitiveness: Technology and the organising of Work, OUP, New York.

Kogut, Bruce; Parkinson, David (1993): The Diffusion of American Organizing Principles to Europe, in: Kogut (1993).

Lamoreaux, Naomi R. (1984): The Great Merger Movement in American Business 1895-1904, CUP, New York

Lamoreaux, Naomi; Sokoloff, Kenneth (1997): Investors, Firms and the Market for Technology, paper presented to the NBER Conference.

Lazonick, William (1991): Business, Organisation and the Myth of the Market Economy, CUP, New York.

Lazonick, William (1993): Industry Clusters versus Global Webs: Organizational Capabilities in the American Economy, Industrial and Corporate Change, vol.2, no.1, pp.1-24.

Livermore, Shaw (1935): The Success of Industrial Mergers, Quarterly Journal of Economics, 50 .

Maddison, Angus (1989): The World Economy in the Twentieth Century, Paris.

Alfred Marshall (ed. Guillebaud) (1961): Principles of Economics, (Variorum edition).

Meyer, Edgar J. (1910): Industrial Stocks as Investments, Annals of the American Academy of Political and Social Science, XXXV, May.

Mishino, Kazuhiro (1997): Learning by New Experience, paper presented to the NBER Conference.

Porter, Michael E. (1990): The Competitive Advantage of Nations, Macmillan, London.

Raff, Daniel; Temin, Peter (1997): Sears Roebuck in the Twentieth Century: Competition, Complementarities, and the Problem of Wasting Assets, paper presented to the NBER Conference.

Reich, Robert (1992): The Work of Nations, Vintage Books, NY.

Rumelt, Richard P. (1974): Strategy, Structure and Economic Performance, Boston, Mass.

Rumelt, Richard P. (1991): How much does industry matter?, Strategic Management Journal, 12.

Samber, Mark (1997): Firm Behaviour and Financial Panic in an Industrial District, paper presented to the NBER conference.

Schmalensee, R. (1985): Do Markets Differ Much? American Economic Review, 75, 2.

Schmitz, Christopher (1995): The World's Largest Industrial Companies of 1912, Business History, October, 85-96.

Shannon, H.A. (1933): The Limited Liability Companies of 1866-1883, Economic History Review, IV.

Stigler, George J. (1965): The Dominant Firm and the Inverted Umbrella, Journal of Law and Economics, 8, 1965, reprinted in Stigler (1968).

Stigler, George J. (1968): The Organisation of Industry, Homewood, Illinois.

Teece, David J. (1993): The Dynamics of Industrial Capitalism: Perspectives on Alfred Chandler's Scale and Scope, Journal of Economic Literature, XXX1, March, pp.199-225.

Tilly, Richard (1982): Mergers, external growth and finance in the development of the largescale enterprise in Germany, 1880-1913, Journal of Economic History, 42.

Usselman, Steven W. (1997): Internalization of discovery by American Railroads, paper presented to the NBER Conference.

Wright, Gavin (1997): The Myth of the American Miracle, paper presented the NBER Conference. 